\title{
Mitochondrial DNA Nucleotide Changes in Cataract and Glaucoma Patients in Senegal
}

\author{
Sokhna Oumou Ba ${ }^{1,}$, Fatimata Mbaye ${ }^{1}$, Matar Ciss ${ }^{1}$, Ndéye Ndoumbé Guéye ${ }^{2}$, Mbacké Sembéne ${ }^{1}$ \\ ${ }^{1}$ Genetic for Gestion of Population team (GENGESPOP), Department of Animal Biology, Faculty of sciences and Technics, Cheikh Anta \\ Diop University, Dakar, Senegal \\ ${ }^{2}$ Ophthalmology Department, Principal Hospital, Dakar, Senegal
}

Email address:

sokhnaoumou.ba@ucad.edu.sn(S.O.Ba), fatimata.mbaye@ucad.edu.sn (F. Mbaye), khoumafr@yahoo.fr(M. Ciss), nng67@hotmail.com (N. N. Guéye), mbacke.sembene@ucad.edu.sn (M. Sembéne)

*Corresponding author

\section{To cite this article:}

Sokhna Oumou Ba, Fatimata Mbaye, Matar Ciss, Ndéye Ndoumbé Guéye, Mbacké Sembéne. Mitochondrial DNA Nucleotide Changes in Cataract and Glaucoma Patients in Senegal. International Journal of Genetics and Genomics. Vol. 6, No. 3, 2018, pp. 30-36.

doi: $10.11648 /$ j.jigg.20180603.11

Received: October 3, 2018; Accepted: October 19, 2018; Published: November 6, 2018

\begin{abstract}
Cataract and glaucoma are the leading causes of blindness in the world. The aim of this study was to investigate the involvement of somatic mutations of the MT-CYB gene in glaucoma and cataract cases among Senegalese patients. 38 samples including 12 controls and 26 patients (18 cataracts, 8 glaucomas) were used to study polymorphism, diversity and genetic structuration of the MT-CYB gene. DNA was isolated from whole blood samples and then the gene was genotyped by PCR-Sequencing. The study of the chromatograms and sequences obtained made it possible to detect a total of twenty-four (24) variants among which nine (9) non-synonymous mutations with seven (7) different positions. Two of them were common to both pathologies (G71A, T96C) and predicted to be non-pathogenic. The insertion, T235TA was specific for glaucoma and is known to be pathogenic. Four (4) mutations were specific to cataract (T118TC, A401AC, G402C and A408AC). T118TC and $\mathrm{A} 408 \mathrm{C}$ are predicted pathogenic. A total of four (4) haplogroups (H, J, L and M) were found in this study. The haplogroups $\mathrm{H}$ and $\mathrm{L}$ are significantly represented in patients with cataract $(\mathrm{H}: \mathrm{p}$-value $=1.72182 \mathrm{e}-09$ and $\mathrm{L}: \mathrm{p}$-value $=0.000351)$ and glaucoma $(\mathrm{H}$ : $\mathrm{p}$-value $=3.333 \mathrm{e}-08$ and $\mathrm{L}$ : $\mathrm{p}$-value $=0.009398)$. The results also revealed a differentiation only between controls and glaucoma patients $(\mathrm{Fst}=0.17144$ and $\mathrm{p}$-value $=0.0019)$ and between controls and glaucomatous belonging to haplogroup L $(\mathrm{Fst}=0.47368$ and $\mathrm{p}$-value $=0.0156)$. The conclusions from this work were that contrary to cataract, MT-CYB somatic mutations are involved in the occurrence of glaucoma in Senegalese and this involvement is correlated with mitochondrial haplogroup L.
\end{abstract}

Keywords: Cataract, Glaucoma, DNA, Mitochondria, MT-CYB Gene

\section{Introduction}

The vision is the most precious of the five senses. According to the World Health Organization [1], in 2010, close to 285 million people were visually impaired, with 39 million of them blind. Ninety percent $(90 \%)$ of people with visual impairments live in low-income countries [1] Cataract is one of the main causes of blindness and visual impairment worldwide, increasing the public health and economic burden of this disease [2]. In 2007, the WHO estimated that cataract was responsible for blindness in thirty-nine percent (39\%) of the world's 37 million blind people. In people aged 50 years and older, the prevalence of cataracts is greater than the combined prevalence of glaucoma and age-related macular degeneration [3]. The rate of blindness by cataract vary considerably from one country to another due to disparities in financial resources, availability of ophthalmologists, perceived need to improve vision, genetic and environmental factors [4]. One of the main difficulties with glaucoma and blindness for which he may be responsible is the lack of a uniform definition of these diseases [1]. Globally, after the non-corrected default of refraction and non-operated cataract, glaucoma $(2 \%)$ is the principal cause of visual deficiency and 
is one of the avoidable causes of blindness [1]. In less developed countries, particularly in sub-saharan Africa, the incidence of glaucoma is $15 \%$ [1]. According to the National Blindness Control Program, the prevalence of blindness in Senegal is $1.4 \%$ and glaucoma is the third main cause of blindness after cataract and trachoma; with a prevalence of $0.14 \%$ [5]. These barriers emphasize the importance of preventing or delaying cataract and glaucoma formation.

Since the advent of molecular biology, researchers have highlighted the implication of genetics in the development of ocular pathologies $[6,7]$ and the mitochondrial genome has in recent years been investigated in relation to these pathologies $[8,9]$. It has been suggested that, similar to other optic nerve atrophies, mitochondrial dysfunction or altered mitochondrial signaling pathways are involved in the glaucoma pathogenesis $[10,11]$. In a recent article on POAG in Saudi Arabia [12], no pathological nucleotide changes were detected for the autosomic causative genes myocilin (MYOC) and optineurin (OPTN). Conversely, the number of nonsynonymous mutations and transversions found in the mitochondrial DNA (mtDNA) coding region of POAG patients was significantly greater than in control subjects. Although no individual mutations could be associated with POAG, these data suggested that mitochondrial dysfunction may be a risk factor for this disease [13]. Therefore, the objective of this study was to analyze the somatic mutations of the MT-CYB gene in cataract and glaucoma cases in Senegal.

\section{Material and Methods}

\subsection{Population Study}

A total of 38 blood samples were collected at the Ophthalmology Department of Aristide Le Dantec Hospital and Principal Hopital in Senegal. The subjects included 18 cataract patients, 8 glaucoma patients and 12 controls. The ethical approval for this study was obtained from the Cheikh Anta Diop University Ethical Committee (Protocol 0269/2018/CER/UCAD).

\subsection{DNA Extraction, PCR and Sequencing of the MT-CYB Gene}

Genomic DNA was extracted from whole-blood samples of all patients and control using the QIAamp DSP DNA Blood Kit (Qiagen) according to the manufacturer's instructions. Amplification of MT-CYB was carried out as described previously by Mbaye et al. (2014) [14]. All the PCR products were purified and sequenced with the ABI Big Dye Terminator cycle sequencing ready reaction kit and an ABIPRISM 3730xl sequencer (Applied Biosystems, Foster City, CA).

\subsection{Molecular Analysis}

The chromatograms obtained after sequencing were submitted to Mutation surveyor software version 5.0 [15] in order to identify the mutations and to determine their nature (homoplasmic or heteroplasmic) and their status (transition or transversion). MtDNA polymorphisms were compared with the mitochondrial genome data base of world population by using Mitomap [16]. Those not found in the Mitomap database were recorded as new mutations. For the Cytochrome $\mathrm{b}$ protein coding gene, three following in silico algorithms were used to predict the putative effect of each nonsynonymous mutation on protein function: Polyphen-2 [17], SIFT [18] and Provean [19]. A mutation was considered pathogenic when:

(1) It was not reported in mitochondrial databases or Medline-listed literature as a confirmed polymorphism;

(2) It was not present in control subjects;

(3) It changed a moderately or highly conserved amino acid;

(4) It occurred in a region of high interspecific conservation;

(5) Provean predicted an alteration of the protein structure;

(6) It was assessed as possibly or probably pathogenic by PolyPhen-2; and

(7) It was predicted by SIFT to have an effect on protein function.

The sequence patterns were observed and edited using BioEdit sequence Alignment Editor V.7.1.9 [20]. MT-CYB sequences were submitted to the MITOMASTER database [21] which uses the Cambridge reference sequence NC_012920.1 to classify them according to the haplogroups. The genetic diversity of the MT-CYB gene between controls and patients was studied by calculating the number of polymorphic sites, the total number of mutations, the frequency of nucleotide composition and the ratio of transition to transversion using DnaSP v 5.10.01 [22] and MEGA7 [23]. The parameters of specific diversity of population (genetic distance and FST) and genetic structuration (AMOVA) were calculated respectively with MEGA 7 [23] and Arlequin [24]. The parameters and report of the differentiation and the structuration of the MTCYB gene were done depending of the population.

\subsection{Statistical Analysis}

The normality of the data was tested under XLSTAT 2018.3.50896 using the Shapiro-Wilk test. The Fisher test with XLSTAT 2018.3.50896 was conducted to detect the correlation between MT-CYB mutations and cataract as well as glaucoma. The frequency of each haplogroup among cases and controls were compared with the X2 test (Fisher's exact test where appropriate) XLSTAT and Mitotool software, to correlate the risk of having cataract or glaucoma with the haplogroups. A 95\% confidence interval (CI) was calculated and $\mathrm{p}<0.05$ was considered statistically significant.

\section{Results}

\subsection{MT-CYB Somatic Mutations}

The mutation results are summarized in Table 1. Twentyfour variants with nine $(37.5 \%)$ transitions and fifteen $(62.5 \%)$ transversions were observed in the study population with twenty-three variants found in the subjects with cataract. Eight $(34.78 \%)$ of the mutations were non-synonymous while fourteen $(62.51 \%)$ were synonymous. Five variants were 
observed among glaucoma patients with three nonsynonymous mutation. Of the seven non-synonymous mutations observed, two (G71A, T96C) were found to be non-pathologic in both cataract and glaucoma. An insertion, (T235TA) was found to be specific to glaucoma and this is known to be pathogenic. Four mutations, (T118TC, A401AC, G402C and A408AC) were specific to cataract while T118TC and $\mathrm{A} 408 \mathrm{C}$ are predicted to be pathogenic.

\subsection{Haplogroups of the MT-CYB}

Out of the thirty-eight genotyped samples, four haplogroups $(\mathrm{H}, \mathrm{J}, \mathrm{L}, \mathrm{M})$ were found. Haplogroup $\mathrm{J}$ was found only in patients with cataracts. Haplogroup $\mathrm{H}$ was absent for control subjects and more represented in cataract patients with the sub-haplogroup H65 which is specific to it while sub haplogroup $\mathrm{H} 2 \mathrm{a}$ is common to both diseases. $44.73 \%$ of the studied population had the haplogroup L with the L2e sub-haplogroup specific to the controls and L2c common among the cataracts, glaucoma and control groups. Haplogroup $M$ was found among the cataract patients (specifically M9a and M24) and controls. $\mathrm{H}$ and L haplogroups were significant at $\mathrm{p}<0.05$ in both cataract and glaucoma patients whereas haplogroup $\mathrm{M}$ was not significant among the groups and was present only in cataract patients.

Table 1. Polymorphism of MT-CYB gene in patients and controls.

\begin{tabular}{|c|c|c|c|c|c|c|c|}
\hline \multirow{2}{*}{ Mutations } & \multirow{2}{*}{ Score } & \multirow{2}{*}{ p. rCRS } & \multicolumn{2}{|c|}{ Pathologies } & \multirow{2}{*}{ Status } & \multirow{2}{*}{ Nature } & \multirow{2}{*}{ p. $\mathbf{A A}$} \\
\hline & & & Cataract $\%$ & Glaucoma\% & & & \\
\hline T61TA & 31 & 15860 & 5.88 & 0 & Hetero & Trv & L371L \\
\hline G71A & 80 & 15851 & 10.52 & $20 *$ & Homo & $\operatorname{Trs}$ & T368I \\
\hline G81A & 96 & 15840 & 10.52 & 0 & Homo & Trs & L365L \\
\hline T96C & 99 & 15824 & $10^{*}$ & $20 *$ & Homo & Trs & $\mathrm{T} 360 \mathrm{~A}$ \\
\hline $\mathrm{T} 118 \mathrm{TC}$ & 63 & 15802 & 5.26 & 0 & Hetero & Trs & Q352W \\
\hline A136G & 109 & 15784 & $26.31^{*}$ & $0 *$ & Homo & Trs & P346P \\
\hline T235TA & 52 & 15684 & $0 *$ & $20 *$ & Hetero & Trv & Q313H \\
\hline A $272 G$ & 20 & 15648 & 5.26 & 0 & Homo & $\operatorname{Trs}$ & L301L \\
\hline A319G & 151 & 15617 & 7.14 & $0 *$ & Homo & Trs & P285P \\
\hline A350T & 89 & 15570 & 7.14 & $0 *$ & Homo & $\operatorname{Trv}$ & L275L \\
\hline A350AT & 37 & 15570 & 7.14 & $0 *$ & Hétéro & Trv & $\mathrm{L} 275 \mathrm{~L}$ \\
\hline $\mathrm{A} 401 \mathrm{AC}$ & 21 & 15519 & 11.11 & $0^{*}$ & Hetero & Trv & L258G \\
\hline G402C & 50 & 15518 & 11.11 & $0 *$ & Homo & Trv & L258G \\
\hline G402GC & 33 & 15518 & 11.11 & $0 *$ & Hetero & Trv & L258G \\
\hline A408C & 72 & 15512 & $22.22 *$ & $0 *$ & Homo & Trv & Y256D \\
\hline A408AC & 60 & 15512 & 11.11 & $0 *$ & Hetero & Trv & Y256D \\
\hline A409T & 22 & 15511 & 12.5 & 0 & Homo & $\operatorname{Trv}$ & $\mathrm{N} 255 \mathrm{~N}$ \\
\hline A409AT & 45 & 15511 & 12.5 & 0 & Hetero & Trv & $\mathrm{N} 255 \mathrm{~N}$ \\
\hline T411TC & 28 & 15509 & 12.5 & 0 & Homo & $\operatorname{Trs}$ & $\mathrm{N} 255 \mathrm{~N}$ \\
\hline A558T & 32 & 15362 & 1 & 0 & Homo & $\operatorname{Trv}$ & L239L \\
\hline A558AT & 53 & 15362 & 0 & 1 & Hetero & Trv & L239L \\
\hline A597AC & 46 & 15324 & 1 & 1 & Hetero & Trv & S193A \\
\hline
\end{tabular}

Table 1. Continue.

\begin{tabular}{|c|c|c|c|c|c|c|}
\hline Mutations & Conserved domains & PolyPhen-2 & Provean & Sift & Conclusion & References \\
\hline T61TA & Yes & & & & & \\
\hline G71A & Yes & Benign & Neutral & Tolerable & Non pathologic & \\
\hline G81A & Yes & & & & & \\
\hline T96C & Yes & Benign & Neutral & Tolerable & Non pathologic & + Breast cancer $(\mathrm{A} / \mathrm{G})$ \\
\hline T118TC & Yes & Prodom & Deleterious & Affected FP & pathologic & \\
\hline A136G & Yes & & & & & +Breast cancer $(\mathrm{T} / \mathrm{G})$ and POAG \\
\hline A199G & Yes & & & & & + Breast cancer $(\mathrm{T} / \mathrm{C})$ \\
\hline T235TA & Yes & Prodom & Neutral & Affected FP & pathologic & \\
\hline $\mathrm{A} 272 \mathrm{G}$ & Yes & & & & & \\
\hline A319G & Yes & & & & & \\
\hline $\mathrm{A} 350 \mathrm{~T}$ & Yes & & & & & \\
\hline $\mathrm{A} 350 \mathrm{AT}$ & Yes & & & & & \\
\hline $\mathrm{A} 401 \mathrm{AC}$ & Yes & Benign & Neutral & Affected FP & Non pathologic & \\
\hline G402C & Yes & Benign & Neutral & Affected FP & Non pathologic & \\
\hline G402GC & Yes & Benign & Neutral & Affected FP & Non pathologic & \\
\hline G404GC & Yes & & & & & \\
\hline A408C & Yes & Prodom & Deleterious & Affected FP & pathologic & \\
\hline A408AC & Yes & Prodom & Deleterious & Affected FP & pathologic & \\
\hline A409T & Yes & & & & & \\
\hline A409AT & Yes & & & & & \\
\hline $\mathrm{T} 411 \mathrm{TC}$ & Yes & & & & & \\
\hline
\end{tabular}




\begin{tabular}{lllllll}
\hline Mutations & Conserved domains & PolyPhen-2 & Provean & Sift & Conclusion & References \\
\hline A558T & Yes & & & & \\
A558AT & Yes & & & & \\
A597AC & No & & & & \\
\hline
\end{tabular}

Non-synonymous mutations are shown in bold; p. rCRS: position on the Cambridge reference sequence NC_012920.1; p. AA: position of the amino acid relative to the reference protein sequence (Uniprot accession number: P00156); Homo: homoplasmic mutation; Hetero: heteroplasmic mutation; Trs: transition; Trv: transversion; Domain Preserved: Yes if the mutation is in one of these domains (1: MT-CYB (cl27766), (bp: 52-570), conserved in mammals; 2: Cytochrom_B_C, (bp: 97-399), preserved in cellular organisms, 3: QcrB, (bp: 97-564), conserved in cellular organisms, 4: cytochrom_b_C, (bp: 115-555), conserved in cellular organisms; 5: Cytb6 / f_IV, (bp: 301 -435), preserved in cellular organisms). Prodom: Probably damaging; Affects FP: Affects the Protein function; POAG: Primitive Glaucoma with Open Angle. *: meaningful fisher test; + : listed in mitomap

Table 2. Frequencies and diversity of haplogroups found in the study population.

\begin{tabular}{llllll}
\hline Haplogroups & Controls & Cataracts & P-values & Glaucomas & P-values \\
\hline H & - & 5 & $1.72182 \mathrm{e}-09^{*}$ & 2 & $3.333 \mathrm{e}-08^{*}$ \\
$\mathrm{~J}$ & - & 1 & $0.043445^{*}$ & - & \\
$\mathrm{L}$ & 7 & 5 & $0.000351^{*}$ & 5 & $0.009398^{*}$ \\
$\mathrm{M}$ & 2 & 3 & 0.235279 & - & \\
\hline
\end{tabular}

*: P-values $<0.05$

\subsection{Genetic Diversity of the MT-CYB}

The $337 \mathrm{bp}$ of the MT-CYB gene analysed in the twentysix patients suffering oculars diseases with eighteen cataracts and eight glaucoma show $5.04 \%$ polymorphic sites for cataract with $29.41 \%$ of them been informative in parsimony. Among the twelve controls subjects, $1.48 \%$ of the sites were polymorphic with a representative informative site of $40 \%$ in parsimony. $2.3 \%$ of the sites were polymorphic for glaucoma with $12.5 \%$ informative in parsimony. The percentage of informative sites in parsimony was higher among the control subjects than patients. The total number of mutations observed among subjects with cataract, glaucoma and controls were 21,8 and 5 respectively. The bases $\mathrm{T}$ and $\mathrm{G}$ are the most common with respectively a percentage of nucleotide frequencies of: $30.30 \%$ and $34.77 \%$ for subjects with cataract, $30.8 \%$ and $33.92 \%$ for glaucoma subject and $30.24 \%$ and 34.69 for the controls subjects. The percentage of transversion mutation observed among subjects with cataract and glaucoma was $50.60 \%$ and $79.73 \%$ respectively while that for control subjects was $2.03 \%$. Therefore, the ratio of transition to transversion of cataract and glaucoma patients was respectively 0.8 and 0.33 . The parameters for the genetic diversity of the 3 populations are stated below in the table 3 .

Table 3. Parameters for genetic diversity of patients and controls.

\begin{tabular}{|c|c|c|c|c|}
\hline & & Controls & Cataracts & Glaucomas \\
\hline \multicolumn{2}{|l|}{ Sample Size $(\mathrm{N})$} & 12 & 18 & 8 \\
\hline \multicolumn{2}{|c|}{ Sequence length without gaps (L) } & 337 & 337 & 349 \\
\hline \multicolumn{2}{|c|}{ Polymorphic sites (V) } & $5(1.48 \%)$ & $17(5.04 \%)$ & $8(2.37 \%)$ \\
\hline \multicolumn{2}{|c|}{ Variable sites informative $(\mathrm{Pi})$} & $2(40 \%)$ & $5(29.41 \%)$ & $1(12.5 \%)$ \\
\hline \multirow{4}{*}{ Percentage of nucleotide } & $\% \mathrm{~T}$ & 30.24 & 30.30 & 30.8 \\
\hline & $\% \mathrm{C}$ & 9.82 & 9.93 & 9.74 \\
\hline & $\% \mathrm{~A}$ & 25.25 & 25.21 & 25.47 \\
\hline & $\% \mathrm{G}$ & 34.69 & 34.77 & 33.92 \\
\hline \multicolumn{2}{|c|}{ Total number of mutations (Eta) } & 5 & 21 & 8 \\
\hline \multirow{2}{*}{ Nature of the mutations } & Trs. s: & 97.97 & 40.40 & 20.27 \\
\hline & Trv, v: & 2.03 & 59.60 & 79.73 \\
\hline \multicolumn{2}{|c|}{ Repport Trs/Trv (R) } & 42.15 & 0.82 & 0.33 \\
\hline \multicolumn{2}{|c|}{ Mean number of nucleotide differences $\mathrm{K}$} & 1.212 & 3.092 & 2.286 \\
\hline
\end{tabular}

\subsection{Differentiation and Genetic Structure of Populations}

The genetic distances (d) that account for the intra and inter-populations genetic difference (Table 4) and the degree of genetic differentiation (Fst) (Table 5) were calculated, first for each population and then for different groups divided into individuals belonging or not to the Haplogroup L. Genetic distances between patients with cataract (0.009) and glaucoma (0.007) were greater than those observed in controls $(0.004)$. This distance is smaller between controls $(0.021)$ and among cataract patients $(0.006)$ belonging to

haplogroup L compared to the distance between controls $(0.038)$ and patients with cataract $(0.011)$. In glaucomatous patients, the genetic distance is zero between individuals not belonging to haplogroup $\mathrm{L}$ and 0.020 in individuals belonging to this haplogroup.

Table 5 shows a genetic differentiation of 0.171 between controls and glaucoma patients $(p$-value $=0.0019)$ but not significant between controls and cataract patients. Based on the haplogroups, a differentiation was noted only between controls and glaucomatous belonging to haplogroup L. 
Table 4. Intra and inter-population genetic distances.

\begin{tabular}{llllll}
\hline Groups & \multicolumn{2}{l}{ Intra genetic distances } & Groups & Intra genetic distances \\
\hline Controls & \multirow{2}{*}{$0.004+/-0.002$} & T 0.038 & Controls-cataract & $0.007+/-0.002$ & TL -CL 0.130 \\
& & TL 0.021 & & & T-C 0.161 \\
Cataracts & $0.009+/-0.003$ & C 0.011 & Controls-glaucoma & $0.006+/-0.002$ & TL-GL 0.128 \\
& & CL 0.006 & & T-G 0.167 \\
glaucomas & $0.007+/-0.002$ & G 0.000 & GL 0.020 & & \\
\hline
\end{tabular}

$\mathrm{T}=$ Controls, $\mathrm{C}=$ Cataracts, $\mathrm{G}=$ Glaucoma, $\mathrm{TL}=$ Controls belonging haplogroup $\mathrm{L} \mathrm{CL}=$ patients with Cataracts belonging haplogroup $\mathrm{L}, \mathrm{GL}=$ patients with Glaucoma belonging haplogroup $\mathrm{L}$

Table 5. Values of genetic differentiation (Fst).

\begin{tabular}{lllllll}
\hline & Groups & & haplogroups & & \\
\cline { 2 - 7 } & Controls-cataracts & Controls-glaucomas & TL-CL & T-C & TL-GL & T-G \\
\hline Fst & 0.0199 & 0.1714 & 0.0359 & 0.0004 & 0.4736 & 0.1540 \\
P Values & 0.1984 & 0.0019 & 0.2942 & 0.3118 & 0.0156 & 0.1163 \\
\hline
\end{tabular}

$\mathrm{TL}=$ Controls belonging haplogroup $\mathrm{L} \mathrm{CL}=$ patients with Cataracts belonging haplogroup $\mathrm{L}, \mathrm{GL}=$ patients with Glaucoma belonging haplogroup $\mathrm{L}$

\section{Discussion}

The analysis of the polymorphism in MT-CYB gene revealed 24 mutations with some in the heteroplasmic state and twelve of them are synonymous mutations which did not lead to changes in amino acids. G15784A which has high potential for association with POAG $[25,26]$ and found in 5 cataract patients has been previously reported in mitomap [27]. Although synonymous mutations are oftenly called "silent mutations," growing evidence shows significant effects of them on transcription, splicing, and mRNA transport (translation) that would alter the phenotype [28]. The seven non-synonymous substitutions found in this study showed two pathologic mutations that are common to both pathologies. G15851A was found in two patients with cataract and glaucoma and led to a change from threonine to isoleucine at position 368. In addition, T15824C was found in two patients with cataracts and glaucoma that also changed threonine to alanine. The three mutations suspected to be pathologic in this study were all specific to one disease. Cataract mutation T15802TC (heteroplasmic) was found in one patient and A15512AC present in two individuals (homoplasmic in one and heteroplasmic in the other). T15802TC led to the replacement of glutamine with tryptophan at position 352 . Other studies reported a change of amino acid at this position [29] with often deleterious effects but they are not listed in mitomap. A15512AC, it led to the replacement of tyrosine with aspartic acid. The similarities between glaucoma and various mitochondrial optic neuropathies have generated interest in the study of mitochondrial function and glaucoma abnormalities. T15684TA which is heteroplasmic and led to the change of glutamine to histidine at position 313 is one suspected pathologic mutation found in one patient. The mechanisms of how mitochondrial abnormalities may endanger the optic nerve remain uncertain [30]. There is, however, increasing evidence that both an activation of glial cells and oxidative stress in the axons may play an important role [31]. The pathologic mutations that are observed in the MT-CYB gene could disrupt the normal activity of the electron transport chain, which could affect the production of ATP and increase the production of reactive oxygen species (ROS) [32].

mtDNA accumulates genetic variants over time, and being quasi-maternal, it undergoes negligible intermolecular recombination. As a consequence, the old variants existing in the human population define the haplogroups that remain geographically or ethnically restricted [33]. In addition to the sub Saharan haplogroup $L$ that is found in this study, the European haplogroups $\mathrm{H}$ and $\mathrm{J}$ and the Asian haplogroup $\mathrm{M}$ were also present. The sub-Saharan haplogroup $\mathrm{L}$ is predominantly represented with a very significant difference between controls and patients with glaucoma and could have an influence in the development of this pathology. This supports the fact that the risk of developing glaucoma [34] is associated with Senegalese belonging to mitochondrial haplogroup L. Also, an earlier study has shown the involvement of sub-haplogroups of $\mathrm{L}$ in the occurrence of glaucoma in the African-American population [27]. A study of the Saudi population [13] revealed a positive association of POAG with African haplogroups (L) and evidence of a protective effect of Eurasian haplogroup $(\mathrm{N})$, but significant associations could not be made between mitochondrial haplogroups and POAG in other studies on Arabs [34], Ghanaians [35] and Northern Europeans [36].

Analysis of the genetic variability showed that all the changes observed in the patients were substitutions with more transversions, $59.60 \%$ among cataracts cases and $79.73 \%$ among patients with glaucoma compared to the controls $(2.03 \%)$. Similar results were obtained by AbuAmero et al. [12] among glaucoma patients who had a high frequency of mtDNA transversion $(25 / 34,73.5 \%)$. According to Sekiguchi et al. (2002) the guanine base has the lowest oxidation potential of the four nucleotides of DNA, making the $\mathrm{G}: \mathrm{C} \rightarrow \mathrm{T}: \mathrm{A}$ or $\mathrm{G}: \mathrm{C} \rightarrow \mathrm{C}: \mathrm{G}$ transversion mutations frequent in the context of oxidative stress. The presence of transversions in mitochondrial DNA in patients with GPAO may be evidence that the mechanisms of damage and repair are involved in the generation of these mutations as part of an early oxidative stress response [12]. According to Roshan et 
al. [38], variations in mitochondrial DNA are commonly found in congenital cataract, but the precise role of such alteration is unclear. Comparing the controls to glaucomatous, a relatively low genetic distance of 0.006 was observed with a significant FST. This is because the mutations found in patients with glaucoma appear to be more pathogenic and thus inducing a molecular difference with the controls.

\section{Conclusion}

It has become clear in recent years that mitochondrial dysfunction is intimately linked to many aspects of ophthalmic diseases. This study, aimed at identifying association with these two pathologies revealed three heteroplasmic mutations: two mutations T15802TC and A15512AC were associated with cataract and T15684TA mutation implicated in glaucoma. Other benign mutations were also observed in addition to synonymous mutations. The genetic diversity of the affected population compared to the controls was studied. Thus, in addition to the diversity of nucleotides noted for all the individuals studied, the results revealed the existence of a genetic differentiation between controls and patients that is more pronounced in glaucomatous patients. These results showed also that patients belonging to the mitochondrial haplogroup $\mathrm{L}$ are significantly more likely to contract glaucoma in Senegal.

\section{Acknowledgements}

The authors would like to thank all donors enrolled in the present study.

\section{References}

[1] World Health Organization. (2012). Global Data on Visual Impairments 2010. WHO/NMH/PBD/12.01.

[2] Javitt, J. C., Wang, F. \& West, S. K. (1996). Blindness due to cataract: epidemiology and prevention. Annu Rev Public Health. 17() , 159-77.

[3] McGwin, G., Khoury, R., Cross, J. \& Owsley, C. (2010). Vision impairment and eye care utilization among Americans 50 and older. Curr Eye Res. Jun; 35(6): 451-8. doi: $10.3109 / 02713681003664931$.

[4] Athanasiov, P. A., Edussuriya, K., Senaratne, T., Sennanayake, S., Sullivan, T., Selva, D. \& Casson, R. J. (2010). Cataract in Central Sri Lanka: Prevalence and Risk Factors from the Kandy Eye Study. Ophthalmic Epidemiology. 17(1), 34-40.

[5] Sarr, B. (2008) Pathologies oculaires courantes. PNLC. 2-20.

[6] Damji, K. F. (1999). Advances in molecular genetics of glaucoma: a perspective for the clinician. Semin Ophthalmol. 14(3): 171-179.

[7] Liu, Y., Munro, D., Layfield, D., Dellinger, A., Walter, J., Peterson, K., Rickman, C. B., Allingham, R. R. \& Hauser, M. A. (2011). Serial analysis of gene expression (SAGE) in normal human trabecular meshwork. Mol Vis. 93, 817-885.
[8] Brennan, L. A. \& Kantorow, M. (2009). Mitochondrial function and redox control in the aging eye: role of MSRA and other repair systems in cataract and macular degenerations. Exp Eye Res. 88, 195-203.

[9] Jarrett, S. G., Alfred, S. L. \& Michael, E. B. (2010). The Importance of Mitochondria in Age-Related and Inherited Eye Disorders. Ophthalmic Res. 44, 179-190.

[10] Osborne, N. N. (2010). Mitochondria: Their role in ganglion cell death and survival in primary open angle glaucoma. Exp Eye Res. 90: 750-7.

[11] Izzotti, A., Longobardi, M., Cartiglia C. \& Sacca, S. C. (2011) Mitochondrial damage in the trabecular meshwork occurs only in primary open-angle glaucoma and in pseudoexfoliative glaucoma. PLoS One. 6: e14567.

[12] Abu-Amero, K. K., Morales, J. \& Bosley, T. M. (2006). Mitochondrial abnormalities in patients with primary openangle glaucoma. Invest Ophthalmol Vis Sci. 47, 2533-2541.

[13] Abu-Amero, K. K., González, A. M., Osman, E. A., Larruga, J. M., Cabrera, V. M. \& Al-Obeidan, S. A. (2011). Susceptibility to primary angle closure glaucoma in Saudi Arabia: the possible role of mitochondrial DNA ancestry informative haplogroups. Mol Vis. 17(), 2171-2176.

[14] Mbaye, F., Dem, A., Fall, M., Diop, G., Mbengue, B., Diallo, R. N., Niang, M. S., Kane, M., Ka, S., Diéye, A. \& Sembène, M. (2014). Genetic Diversity of Breast Cancer in Senegalese Women: New Insight from Somatic Mutations. Journal of Health Science. 4 (2), 25-33.

[15] https://softgenetics.com/mutationSurveyor.php.

[16] https://www.mitomap.org/foswiki/bin/view/MITOMAP/Poly morphismsCoding

[17] Adzhubei, I., Jordan, D. M. \& Sunyaev, S. R. (2013). Predicting functional effect of human missense mutations using polyPhen-2. Current Protocols in Human Genetics. Chapter 7, (7-20).

[18] Kumar, P., Henikoff, S. \& Ng, P. C. (2009). Predicting the effects of coding non-synonymous variants on protein function using the SIFT algorithm. Nature Protocols. 4(7), 1073-1081.

[19] Choi, Y., Sims, G. E., Murphy, S., Miller, J. R. \& Chan, A. P. (2012). Predicting the functional effect of amino acid substitutions and indels. PloS one, 7, e46688.

[20] Hall, T. (1999). BioEdit version 7.1.9. Department of Microbiology, North Carolina State University.

[21] Lott, M. T., Leipzig, J. N., Derbeneva, O., Xie, H. M., Chalkia, D., Sarmady, M., Procaccio, V. \& Wallace D. C. (2009). MITOMASTER - A Bioinformatics Tool For the Analysis of Mitochondrial DNA Sequences. Hum Mutat. 2009 Jan; 30(1): 1-6.

[22] Rozas, J., Librado, P., Sánchez-Del Barrio, J. C., Messeguer, X. \& Rozas, R. (2010). DnaSP Version 5 Help Contents [Help File]. Available with the program at http://www.ub.edu/dnasp/.

[23] Tamura, K., Stecher, G., Peterson, D., Filipski, A. \& Kumar, S. (2014). "MEGA 7: Molecular Evolutionary Genetics Analysis using Maximum Likelihood, Evolutionary Distance, and Maximum Parsimony Methods". Molecular Biology and Evolution. 30, 2725-2729. 
[24] Excoffier, L., Laval, G. \& Schneider, S. (2005). Arlequin version. 3.0: An integrated software package for population genetics data analysis. Evolutionary Bioinformatics Online. 1, $47-50$

[25] Astafurov, K., Elhawy, E., Ren, L., Dong, C. Q., Igboin, C., Hyman, L., Griffen, A., Mittag, T. \& Danias, J. (2014). Oral microbiome link to neurodegeneration in glaucoma. PLoS One. 9(9), 104-416.

[26] Ma, J., Coarfa, C., Qin, X., Bonnen, P. E., Milosavljevic, A., Versalovic, J. \& Aagaard, K. (2014). mtDNA haplogroup and single nucleotide polymorphisms structure human microbiome communities. BMC Genomics. 15(), 257.

[27] Collins, D. W., Gudiseva, H. V., Trachtman, B., Bowman, A. S., Sagaser, A., Sankar, P., Miller-Ellis, E., Lehman, A., Addis, V. \& O'Brien, J. M. (2016). Association of primary open-angle glaucoma with mitochondrial variants and haplogroups common in African Americans. Mol Vis. 22 (), 454-471.

[28] Goymer, P. (2007). Synonymous mutations break their silence. Nat Rev Genet. 8, 92.

[29] http://mitimpact.css-mendel.it

[30] Howell, N. (2003). LHON and other optic nerve atrophies: the mitochondrial connection. Dev Ophthalmol.37, 94-108.

[31] Tezel, G. \& Wax, M. B. (2003). Glial modulation of retinal ganglion cell death in glaucoma. JGlaucoma.12(1): 63-8.

[32] West A. P., Shadel G. S. \& Ghosh S. (2011). Mitochondria in innate immune responses. Nat Rev Immunol. 11(6): 389-402.
[33] Kenney, M C., Chwa, M., Atilano, S R., Falatoonzadeh, P., Ramirez, C., Malik, D., Tarek, M., Del Carpio, J. C., Nesburn, A. B., Boyer, D. S., Kuppermann, B. D., Vawter, M. P., Jazwinski, S. M., Miceli, M. V., Wallace, D. C. \& Udar, N. (2014). Molecular and bioenergetic differences between cells with African versus European inherited mitochondrial DNA haplogroups: implications for population susceptibility to diseases. Biochim Biophys Acta. 1842(2), 208-219.

[34] Abu-Amero, K. K., Morales, J., Bosley, T. M., Mohamed, G. H. \& Cabrera, V. M. (2008). The role of mitochondrial haplogroups in glaucoma: a study in an Arab population. Mol Vis. 14()$, 518-522$.

[35] Abu-Amero, K. K., Hauser, M. A., Mohamed, G., Liu, Y., Gibson, J., Gonzalez, A. M., Akafo, S. \& Allingham, R. R. (2012). Mitochondrial genetic background in Ghanaian patients with primary open-angle glaucoma. Mol Vis. 18(), 1955-1959.

[36] Andrews, R., Ressiniotis, T., Turnbull, D. M., Birch, M., Keers, S., Chinnery, P. F. \& Griffiths, P. G. (2006). The role of mitochondrial haplogroups in primary open angle glaucoma. Br J Ophthalmol. 90(4), 488-490.

[37] Sekiguchi, M. \& Tsuzuki, T. (2002). Oxidative nucleotide damage: consequences and prevention. Oncogene. 21: 88958904.

[38] Roshan, M., Kabekkodu, S. P., Vijaya, P. H., Manjunath, K., Graw, J., Gopinath, P. M. \& Satyamoorthy, K. (2012). Analysis of mitochondrial DNA variations in Indian patients with congenital cataract. Mol Vis. 18, 181-193. 